\title{
Die Nase und nicht die Hautreaktion behandeln!
}

I $\mathrm{n}$ der industrialisierten Welt leiden ca. $15 \%$ der Bevölkerung unter chronischen Nasenbeschwerden, die von den Mitmenschen gerne banalisiert werden, die Betroffenen aber sehr stören und in ihrer Lebensqua-

\section{"To deal with the nose is to ask for problems."}

Niels Mygind lich mehr Patienten, bei denen die allergische Komponente im Vordergrund steht. Aber selbst dann gilt es zu bedenken, dass ein großer Teil der Beschwerden nicht mehr tatsächlich durch die Allergenexposition ausgelöst wird, sondern durch die lität einschränken können. Alles allergisch? Wenn es nur so einfach wäre!

Die möglichen Ursachen chronischer Rhinopathien sind sehr vielfältig und oft nur mit großem diagnostischem Aufwand mit Sicherheit festzustellen. Was in der täglichen Praxis des NichtFacharztes oft innerhalb von drei Minuten in die Gruppen „allergisch“ oder „vasomotorisch“ unterteilt wird, läßt sich in einem rhinologischen Zentrum mittels einer komplexen Diagnostik in eine Vielzahl völlig unterschiedlicher pathophysiologischer Störungen unterteilen. Und auch hier bleibt ein „idiopathischer" Rest.

\section{Überbewertung perennialer Allergien}

Die von A. Riechelmann an der Universitätsklinik Ulm erhobenen Daten von 2.194 Patienten mit einer chronischen Rhinopathie sind beeindruckend ( $S$. 386 in diesem Heft). Auch wenn etwa ein Drittel dieser Patienten einen positiven Hauttest auf ein perenniales Allergen aufweist, so sind es noch nicht einmal 5\%, bei denen die Allergie tatsächlich den Krankheitsauslöser darstellt! Die Überbewertung perennialer Allergien läßt sich nur durch die Durchführung des nasalen Provokationstestes vermeiden, der gerade vor der Einleitung therapeutischer Maßnahmen bzw. einer Hyposensibilisierung unverzichtbar erscheint. Es gilt, die Nase und nicht die Hautreaktion zu behandeln!

In der täglichen Routine des allergologisch tätigen Arztes sind es sicherder Schleimhaut. Auch bei diesen komplexen Beschwerdebildern können allergenspezifische Behandlungsmaßnahmen nur einen Teilerfolg erzielen.

\section{„Kochrezept“ für den nasalen Provokationstest}

Der nasale Provokationstest ist also unverzichtbar bei der Diagnostik perennialer Rhinopathien. Er wurde vor mehr als 125 Jahren von Blackley eingeführt und wird heute trotz der inzwischen erreichten Standardisierung durch einen Arbeitskreis der DGAI vor zehn Jahren noch immer in allen möglichen Modifikationen durchgeführt. International ist der Test beinahe ausschließlich für wissenschaftliche Ansätze gebräuchlich, auch wenn in letzter Zeit mehr Interesse an einer Standardisierung für den Praxisgebrauch zu erkennen ist. Mit Recht können wir behaupten, dass diese Standardisierung des nasalen Provokationstestes in Deutschland international vorbildlich ist. Der heutige Stand der Empfehlungen wird Ihnen in dieser Ausgabe des ALLERGO JOURNALS ,als Kochbuch zum Nachmachen“ dargestellt (S. 396).

\section{Neidischer Blick auf die Pneumologen?}

Ein Problem bleibt allerdings die Diagnose einer individuellen nasalen $\mathrm{Hy}$ perreaktivität, weswegen wir HNOÄrzte die Kollegen aus der Pneumologie beneiden (S. 406 in diesem Heft). Der

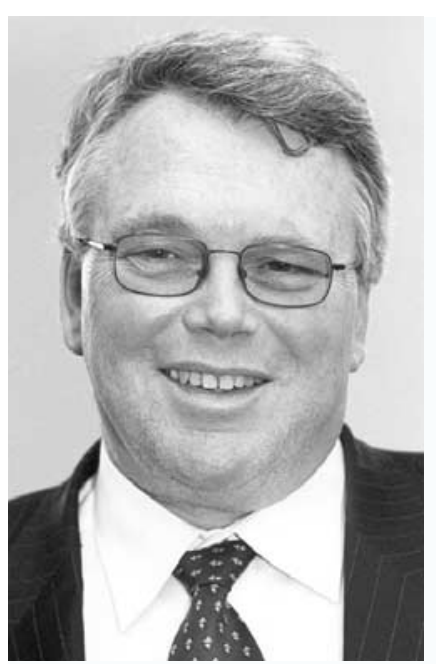

Prof. Dr. Claus Bachert, Universität Gent, hat als Gast-Herausgeber den OriginaliaTeil dieser Ausgabe des ALLERO JOURNALS zum Thema „Diagnostik der allergischen Rhinitis“ betreut. diesem Editorial vorangestellte Satz von Niels Mygind, einem Kenner der Materie, rührt von dieser Schwierigkeit. Histamin gilt bislang als der beste Mediator zur Diagnose einer unspezifischen Hyperreaktivität und kann zumindest Gruppen von Patienten mit hyperreaktiver Nasenschleimhaut von solchen ohne Hyperreaktivität unterscheiden. Für den individuellen Patienten erlaubt dies allerdings nur bei deutlich gesteigerter Reaktion eine eindeutige Aussage.

Hier besteht also durchaus noch Forschungsbedarf, den ich den wenigen in Deutschland tätigen rhinologischen Zentren gerne ans Herz legen möchte!
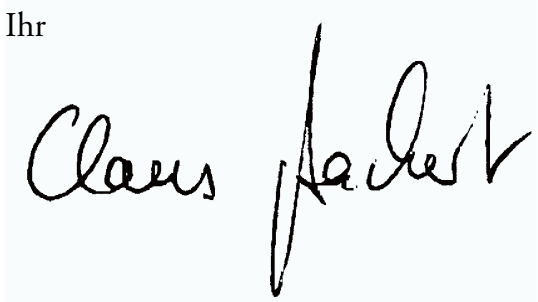

Prof. Dr. C. Bachert 